\title{
Review of the Innovative Design of Coils for Electromagnetic Forming Process
}

\author{
Awab Elsayed ${ }^{1 *}$, Mohammed Elamin ${ }^{2}$ \\ ${ }^{1}$ TU Dortmund University, Dortmund, Germany \\ ${ }^{2}$ Department of Mechanical and Aerospace Engineering, North Carolina State University, Raleigh, United States \\ *Corresponding author: awab.elsayed1994@gmail.com
}

\begin{abstract}
This review article aims to deliver a full and comprehensive understanding of the different types of coils including their limitations and methods of improvement. The efficient design of coils has a significant impact on enhancing the quality of the poor formability alloys. This study further discusses the role of the most important parameters that play a vital role in coils design. The scope of this review includes the modern design of coils that are used in several industries alongside their most suitable applications and end products. Moreover, based on the findings of some important studies in the literature, a detailed review on the numerous methods to evaluate coils performance and how to improve coils quality is provided.
\end{abstract}

Keywords: Coil components, Coil design, Coils types, Electromagnetic forming.

\section{Introduction}

The demand for lightweight products in most engineering applications urges designers to improve their work to achieve the desired weight while maintaining high strength and reliability. Electromagnetic (EM) forming technique is introduced to enhance the poor formability of aluminum alloys [1]. This technique was proved to be successful in aerospace and automotive industries due to the extensive use of aluminum alloys in those fields [2].

Electromagnetic forming is a high-speed forming method that utilizes electromagnetic force fields to achieve the desired final products [3].

Previous studies showed that electromagnetic forming has many benefits over the conventional forming methods. Using electromagnetic forming manufacturers can typically obtain ten times higher formability than conventional forming [4]. Furthermore, electromagnetic forming produces surfaces with very high quality as no contact is required between the tool and the workpiece [5].

As the findings of Psyk et al. [6] suggests, using electromagnetic forming would expand the applications of low formability alloys at room temperature. Electromagnetic forming ameliorates spring back control and minimizes surface wrinkles. Furthermore, because of Lorentz forces, electromagnetic forming is used to fabricate sheet metals and tubes with different geometries in a wide range of length [7]. It is shown in [7] that a workpiece of $1 \mathrm{~m}$ in length can efficiently be deformed into the desired shape.

It is worth mentioning that electromagnetic forming is very common for finite parts and small features of the large products. However, the success of electromagnetic forming heavily relies on the coil ability to generate the energy required to form the workpiece. The first section of this article is devoted for coils design and development reviews. This discussion also includes the process and end products improvement methods. The second part of this article focuses on the limitation of coils, processes and how to optimize the performance of the coils. The last two sections in this paper review the design configurations that are commonly used, and the current new designs to achieve the desired needs in the industry.

\section{Coils Optimization Electromagnetic Forming}

Ensuring high strength and energy is vital in coils selection as stated in Psyk et al. [6]. While a small fraction of the discharge energy is transmitted to the workpiece, the rest energy is dissipated in the form of heat which significantly damages the coil as shown by Werdelmann et al. [8]. The aforementioned energy behavior is a big challenge in the coil design process. Cao et al. [9] study suggested that more research efforts must be put to fully understand the rise of coil temperature during energy transmission process in electromagnetic forming. The new design of coils should implement these research-based suggestions to resolve the temperature issue and to improve the electromagnetic force distribution.

Optimizing the durability and strength of the coil is important to overcome the deformation reaction force. If the coil is close to the workpiece, this would ensure better deformation on the workpiece. However, this can result in a higher reaction force.

In addition, different values and loads are induced due to the variable gap width during the electromagnetic forming process. This is discussed in more details in [6].

To design an effective coil system, there are two approaches in the literature. The first approach is to make a coil that is durable and can be used for multiple pulses. However, there is a lack of information about this approach. The second approach is to design a single-use coil as in [10]. The coil design must be optimized and tailored to the desired application. For instance, 
the design requirements of a constant-diameter tube are different from those of a variable-diameter tube.

\section{Common Coil Designs in Electromagnetic Forming in Different Applications}

This section covers different types of coil designs and their most relevant applications and how does coil design affect the electromagnetic forming process and the final products.

Psyk et al [6] proposed various designs of coils that ae used in metal forming. In particular, the helical coil is the most common type as it suits the expansion and compression applications. Spiral coil, a coil with several layers and one turn is typically used to form symmetric sheet metals.

Other kinds of coils are discussed in [6] such as trident, ellipsoidal, super ellipsoidal geometries. Flat coils are mostly used in bulk formation process. In this process the coil surface must match the workpiece surface and the gap between the coil and workpiece should be very small. Fig. 1, shows the geometry of the flat and spiral coils.

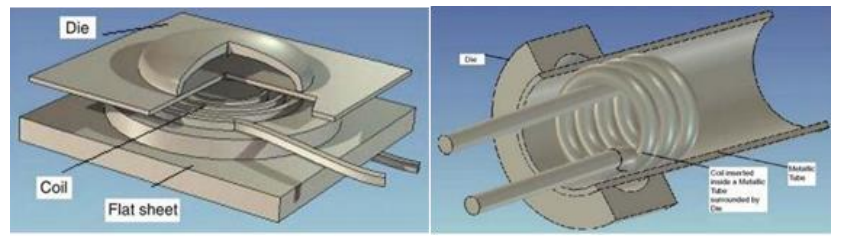

Fig. 1. Flat coil utilized to produce sheet metal and a spiral coil used for tube expansion [11]

Oliveira et al. [12] suggested a box-like double spiral coil to produce a workpiece which cannot be produced in the single spiral coils due to the dead spot at the winding center in the single spiral coil. Ahmed et al. [14] proposed a method to design a coil system with variable sizes and spaces using finite element analysis (FEA) techniques. This method allows the generation of the maximum force and electromagnetic force distribution. FEA showed that increasing the pressure at the center of the workpiece would lead to an improved deformation behavior.

Li et al. [14] introduced the gradient electromagnetic forming (GAMF) to avoid poor dimensional accuracy of variablediameter tubes in constant spacing coils electromagnetic forming process. A mathematical formula was developed by $\mathrm{Li}$ et al. [14] to estimate the coils spacing and electromagnetic force.
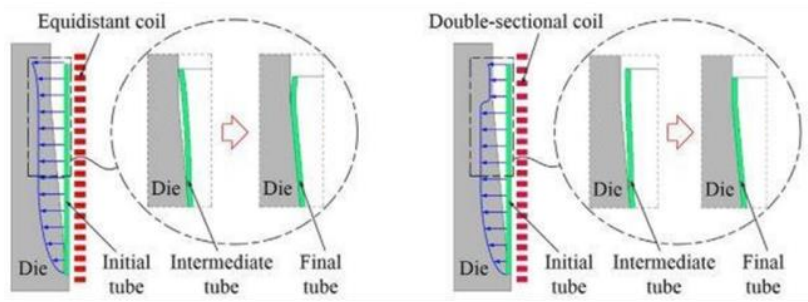

Fig. 2. Forming process in terms of constant spacing coil and doublesectional coil [11]
This will control the force and help produce variablediameter tubes. Furthermore, the method in [14] clearly identified the influence of some parameters such as spacing and die-fitting gap on the coil design. Fig. 2. depicts the forming process of variable diameter tubes with spacing coils and double sectional coil with the GAMF process.

Qiu et al. [15] simulated the tube expansion process using a concave coil in a finite element model. Results indicate that concave coils produce more homogenous axial deformations than the cylindrical coils. Results also showed the existence of two peak electromagnetic forces in addition to the deformation reduction in the middle point.

Using multi-layer coil was proposed by Luo et al. [16] to enhance the depth on the workpiece surface as a result of the equivalent inductance which would eventually increase the energy transmission. The findings in [16] indicated that the multi-layer system is more suitable for large and thick-walled structures.

Furthermore, due to the multi-layer nature, the coils pressure can be uniformly distributed. Also, decreasing the coil distance resulted in a significant energy efficiency due to enhanced electromagnetic force on the workpiece. The components of electromagnetic forming system are shown in fig. 3 .

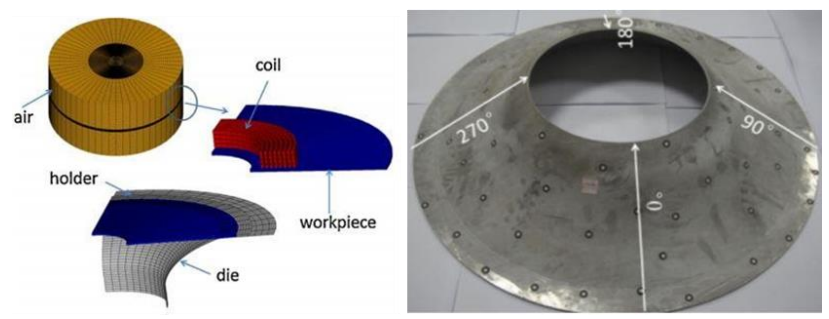

Fig. 3. Multi-layer coil system left) and the finished structure from twotiming EM forming [16]

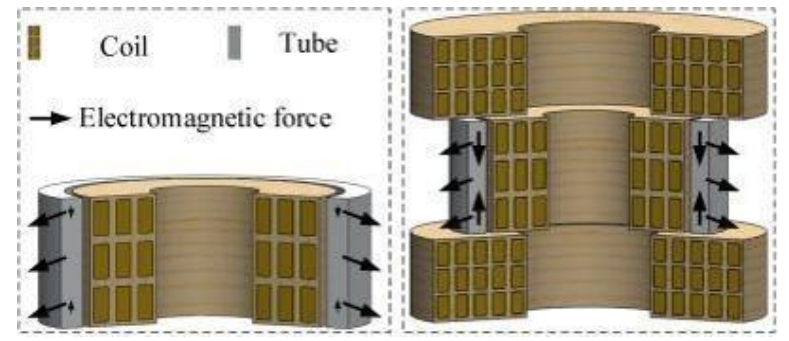

Fig. 4. Comparison between conventional Electromagnetic forming (left), and the enhanced EM with the axial compression coil (right) [15]

Typical electromagnetic forming utilizes spiral coils in tubes expansion. However, this leads to a decreased strength. To solve this issue of the resulting reduced strength, modern designed adopted the idea of using an extra coil in the axial direction to generate an electromagnetic field. The aforementioned technique leads to the expansion and compression of the tube from the radial and axial electromagnetic fields, respectively. More details on this process are provided in [15]. Fig. 4, illustrates the coils in axial and radial direction. 


\section{Optimization Methods of the Electromagnetic Forming Process}

Electromagnetic incremental forming has been introduced as a new innovative process. It simply adds a digital controller to the electromagnetic forming to make it more efficient when forming large components.

The way electromagnetic incremental forming works is by deforming a finite region in the large component, then a computer-controlled system moves the coil on the large sheet.

A multi-layer multi-turn coil is designed considering the strength, inductance, and capacity coefficient [3]. The findings suggested that a higher electromagnetic force was generated from this system in comparison to a similar coil with a single turn.

Lai et al. [7] further analyzed the influence of coil polarity on the current passing through the coils. Lie et al [7] further conducted modeling and experiments on dual coil systems. It is found that the polarity has a significant impact on formability depth, surface smoothening, and energy efficiency.

The workpiece deformation is complex as it relies on some factors such as electromagnetic fields interaction, coilworkpiece interaction. Both interactions affect the flow of the current in the coils and consequently the deformation behavior of the coil. Su et al. [2] showed that the electromagnetic calibration is essential to achieve high dimensional accuracy.

A method that combines forming and calibration is known as "two-step electromagnetic forming". It is used to make oblique holes in the workpiece. On the one hand, experiments are conducted to evaluate the influence of the main process's parameters. On the other hand, simulations are run to analyze the deformation behavior. This method greatly enhances the high dimensional accuracy while reducing the die-fitting gap.

Additionally, to further enlarge the deformation depth, a technique called "time-controlled multi-stage pulsed magnetic field forming" has been introduced. This method also utilizes two coils in the axial and radial directions to produce electromagnetic fields. Higher formability is achieved compared to the traditional single coil forming process.

Fig. 5. illustrates a schematic drawing of a system spacetime-controlled multi-Stage pulsed magnetic field forming.

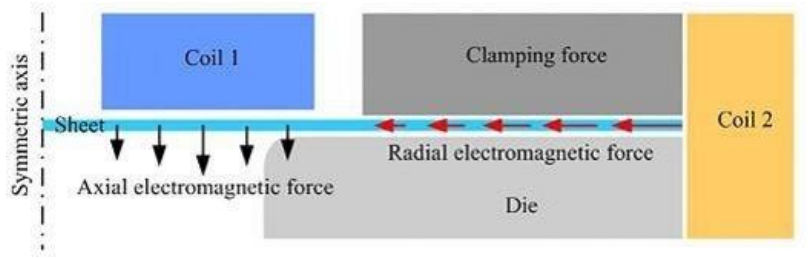

Fig. 5. System space-time-controlled multi-Stage pulsed magnetic field forming [15]

An innovative technique known as electromagnetic-assisted stamping was explained by Okoye et al. [11]. This method focuses on improving the final product quality and ensures high dimensional accuracy of the corner filling. The electromagnetic coils are added to a stamping process. Fig. 6. shows the electromagnetic stamping equipment.
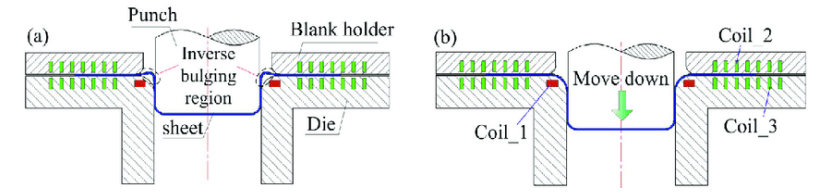

Fig. 6. Electromagnetic-assisted stamping device [17]

Lai et al. [18] proposed a design for the electromagnetic forming system with a double-coil system and utilized it for the purpose of deep drawing. They [18] proved that their system can provide almost twice forming depth of the traditional single coil electromagnetic forming deep drawing process. Furthermore, Lai et al. [18] concluded that the radial force generated from the double coil system can help reduce the tearing effect as a result of the increase in the pressure.

The idea of designing a triple-coil system was investigated by Zhang et al. [19]. They [19] proposed a numerical analysis aiming to increase the deformation depth in the tubing system. This was done by attaching two coils at the two ends of the tube to generate an electromagnetic field.

\section{Summary and Conclusion}

This goal of this review article was to provide a detailed explanation of the multiple coils' types and other discussions on their limitations and methods of improvement. The accurate design of coils in electromagnetic forming is imperative to obtain higher quality of the poor formability alloys. This work investigated the role of the governing parameters that play a significant role in coils design. This review included discussion about the new design of coils that are used in several areas and their typical applications and final products. Furthermore, based on the outcome of several studies provided in the literature, a detailed review on the different methods to evaluate the coils performance and quality was prepared.

\section{References}

[1] Kleiner, M., M. Geiger, and A. Klaus. "Manufacturing of lightweight components by metal forming." Magnesium 530 (2003): 26-66.

[2] Su, Hongliang, et al. "Two-step electromagnetic forming: A new forming approach to local features of large-size sheet metal parts." International Journal of Machine Tools and Manufacture 124 (2018): 99-116.

[3] Qiu, Li, et al. "Design and experiments of a high field electromagnetic forming system." IEEE transactions on applied superconductivity 22.3 (2011): 3700504-3700504.

[4] Chu, Y. Y., et al. "Determination of the flow curve at high strain rates using electromagnetic punch stretching." Journal of Materials Processing Technology 212.6 (2012): 1314-1323.

[5] Huang, Lantao, et al. "Effect of electromagnetic ring expansion on the mechanical property of A5083 aluminum alloy." IEEE transactions on applied superconductivity 24.3 (2013): 1-4.

[6] Psyk, Verena, et al. "Electromagnetic forming — a review." Journal of Materials Processing Technology 211.5 (2011): 787-829.

[7] Lai, Zhipeng, et al. "The effect of coil polarity on electromagnetic forming using a multi-coil system." The International Journal of Advanced Manufacturing Technology 103.1-4 (2019): 1555-1566.

[8] Werdelmann, P., et al. "Assessing the effective energy for magnetic forming processes by means of measurements and numerical calculation." 
Proceedings of the 3rd International Conference on High Speed Forming, Dortmund, Germany. 2008.

[9] Cao, Quanliang, et al. "Analysis and reduction of coil temperature rise in electromagnetic forming." Journal of Materials Processing Technology 225 (2015): 185-194.

[10] Woodward, Steven, et al. "Production of low-volume aviation components using disposable electromagnetic actuators." Journal of Materials Processing Technology 211.5 (2011): 886-895.

[11] Okoye, C. N., J. H. Jiang, and Z. D. Hu. "Application of electromagneticassisted stamping (EMAS) technique in incremental sheet metal forming." International Journal of Machine Tools and Manufacture 46.11 (2006): 1248-1252.

[12] Oliveira, D. A., et al. "Electromagnetic forming of aluminum alloy sheet: free-form and cavity fill experiments and model." Journal of Materials Processing Technology 170.1-2 (2005): 350-362.

[13] Ahmed, M., et al. "Alternative flat coil design for electromagnetic forming using FEM." Transactions of Nonferrous Metals Society of China 21.3 (2011): 618-625.

[14] Li, Jianjun, et al. "Gradient electromagnetic forming (GEMF): a new forming approach for variable-diameter tubes by use of sectional coil."
International Journal of Machine Tools and Manufacture 135 (2018): 6577.

[15] Qiu, $\mathrm{Li}$, et al. "Analysis of electromagnetic force and deformation behavior in electromagnetic forming with different coil systems." International Journal of Applied Electromagnetics and Mechanics 57.3 (2018): 337-345.

[16] Luo, Wenyong, et al. "A novel multi-layer coil for a large and thickwalled component by electromagnetic forming." Journal of Materials Processing Technology 214.11 (2014): 2811-2819.

[17] Cui, Xiaohui, et al. "Deep drawing of cylindrical cup using incremental electromagnetic assisted stamping with radial magnetic pressure." Procedia Engineering 81 (2014): 813-818.

[18] Lai, Zhipeng, et al. "Radial Lorentz force augmented deep drawing for large drawing ratio using a novel dual-coil electromagnetic forming system." Journal of Materials Processing Technology 222 (2015): 13-20.

[19] Zhang, Xiao, et al. "Application of triple-coil system for improving deformation depth of tube in electromagnetic forming." IEEE Transactions on Applied Superconductivity 26.4 (2016): 1-4. 